\title{
Large-momentum tail of one-dimensional Fermi gases with spin-orbit coupling
}

\author{
Fang Qin (覃昉)๑, 1,2,* Pengfei Zhang $\odot,{ }^{3,4}$ and Peng-Lu Zhao ${ }^{1}$ \\ ${ }^{1}$ Shenzhen Institute for Quantum Science and Engineering and Department of Physics, Southern University of Science and Technology \\ (SUSTech), Shenzhen 518055, China \\ ${ }^{2}$ CAS Key Laboratory of Quantum Information, University of Science and Technology of China, Chinese Academy of Sciences, \\ Hefei, Anhui 230026, China \\ ${ }^{3}$ Walter Burke Institute for Theoretical Physics, California Institute of Technology, Pasadena, California 91125, USA \\ ${ }^{4}$ Institute for Quantum Information and Matter, California Institute of Technology, Pasadena, California 91125, USA
}

(Received 1 May 2020; accepted 19 May 2020; published 15 June 2020)

\begin{abstract}
We study the contacts, large-momentum tail, radio-frequency spectroscopy, and some other universal relations for an ultracold one-dimensional (1D) two-component Fermi gas with spin-orbit coupling (SOC). Different from previous studies, we find that the $q^{-8}$ tail in the spin-mixing (off-diagonal) terms of the momentum distribution matrix is dependent on the two SOC parameters in the laboratory frame for 1D systems, where $q$ is the relative momentum. This tail can be observed through time-of-flight measurement as a direct manifestation of the SOC effects on the many-body level. Besides the traditional $1 \mathrm{D}$ even-wave scattering length, we find that two new physical quantities must be introduced due to the SOC. Consequently, two new adiabatic energy relations with respect to the two SOC parameters are obtained. Furthermore, we derive the pressure relation and virial theorem at short distances for this system. To find how the SOC modifies the large-momentum behavior, we take the SOC parameters as perturbations since the strength of the SOC should be much smaller than the corresponding strength scale of the interatomic interactions. In addition, by using the operator product expansion method, we derive the asymptotic behavior of the large-momentum distribution matrix up to the $q^{-8}$ order and find that the diagonal terms of the distribution matrix include the contact of traditional 1D even-wave scattering length as the leading term and the SOC modified terms beyond the leading term, the off-diagonal term is beyond the subleading term and is corrected by the SOC parameters. We also find that the momentum distribution matrix shows spin-dependent and anisotropic features. Furthermore, we calculate the momentum distribution matrix in the laboratory frame for the experimental implication. In addition, we calculate the high-frequency tail of the radio-frequency spectroscopy and find that the presence of the contact related to the center-of-mass momentum in the radio-frequency spectral is due to the SOC effects. This paper paves the way for exploring the profound properties of many-body quantum systems with SOC in one dimension.
\end{abstract}

DOI: 10.1103/PhysRevA.101.063619

\section{INTRODUCTION}

In the strong-coupling regime of cold atomic gases, a series of exact universal relations was established which set up a bridge between the microscopic short-distance correlations and the macroscopic thermodynamic properties of the many-body quantum system [1-7]. These relations show that many thermodynamic properties are connected by a series of universal contact parameters which contain the information of the interaction effect in the large-momentum limit, and they are named contacts. These relations have already been successfully verified in experiments near the $s$-wave Feshbach resonance [8-11]. Furthermore, the universal relations were also studied in other atomic systems, such as the quantum gases with higher-partial-wave interactions [12-19], in one dimension [20-24] and two dimensions [25-32] with threebody correlations [33-39] near a Raman-dressed Feshbach resonance [40] and with ultracold polar molecules [41]. Besides, the contact tensor was predicted in the axisymmetry-

\footnotetext{
*qinfang@ustc.edu.cn
}

broken $p$-wave Fermi gases [42]. At the same time, Zhang et al. predicted the contact matrix and studied the direct connection between the contact matrix and the order parameter of a superfluid [43]. The nuclear neutron-proton contact was introduced in nuclear physics [44], and the general nuclear contact matrices were defined [45] in the context of generalized contacts for realistic interactions [46-50]. There are also other works using Coulomb [51] and realistic atom-atom interactions [52] with the generalized contact formalism.

The successful experimental realization of the synthetic coupling between atomic (pseudo) spin and momentum is another important progress in cold atomic gases [53-59]. This synthetic coupling is so-called spin-orbit coupling (SOC). In one dimension, there is only one type of SOC: The spin is only coupled to the motion of atoms along one spatial direction which is induced by two contour-propagating Raman beams [60-63]. This type of SOC has been realized in experiments for both Bose and Fermi gases [53-57]. Importantly, the SOC can strongly affect the many-body properties $[64,65]$.

Recently, Peng et al. studied the universal relations for the Fermi gases with a three-dimensional isotropic SOC [66]. At the same time, the universal relations for the 
three-dimensional ultracold gases with an arbitrary type of SOC have been investigated [67,68]. Furthermore, the universal relations for spin-orbit-coupled Fermi gases in two dimensions have been derived [69]. However, the one-dimensional (1D) Fermi gases with SOC has not been studied. Here, we investigate the 1D Fermi gases with SOC. Different from the previous studies, we derive the asymptotic behavior of the large-momentum distribution matrix up to the $q^{-8}$ order ( $q$ is the relative momentum) and find that the $q^{-8}$ tail in the spinmixing (off-diagonal) terms of the momentum distribution matrix is dependent on the SOC parameters in the laboratory frame for $1 \mathrm{D}$ systems. This tail can be observed through time-of-flight measurement, and it is a direct manifestation of the SOC effects on the many-body level.

In this paper, we focus on a many-body quantum system that exhibits universal properties, i.e., the 1D two-component Fermi gas with SOC near a broad $s$-wave Feshbach resonance. Such a $1 \mathrm{D}$ system can be realized by applying tight transverse confinements to three-dimensional gases near $s$ wave Feshbach resonances, such as in the fermions of ${ }^{6} \mathrm{Li}$ and ${ }^{40} \mathrm{~K}$ [70-78]. First of all, we give the definition of the traditional even-wave contact for this system. After that, we derive the universal relations for the spin-orbit-coupled Fermi gases in one dimension, including the adiabatic relations, pressure relation, and viral theorem. Besides the traditional even-wave scattering length, we find that two new physical quantities need to be introduced due to the SOC. Using the operator product expansion (OPE) method, we derive the large-momentum tail of the momentum distribution matrix. The momentum distribution matrix shows spin-dependent and anisotropic features. We find that the diagonal elements of the distribution matrix include the contact of the traditional even-wave scattering length as the leading term and the SOC modified terms beyond the leading term, the off-diagonal term is beyond the subleading term and is corrected by the SOC parameters. Furthermore, in order to discuss the experimental implications, we calculate the momentum distribution in the laboratory frame and find that the spin-mixing (off-diagonal) elements of the momentum distribution matrix at large momentum is directly modified by the SOC parameters and the tails which beyond the leading-order term have been changed, the effects can be captured by a time-of-flight measurement in experiments. In addition, we derive the high-frequency radio-frequency spectroscopy and find that the presence of the contact related to the center-of-mass momentum in the radio-frequency spectral is due to the SOC effects.

The paper is organized as the following: In Sec. II, we give the model Hamiltonian. In Sec. III, we calculate the two-body physics. In Sec. IV, we give the definition of the traditional even-wave contact. Furthermore, we study some of the universal relations in Sec. V. Moreover, we calculate the large-momentum distribution tail in Sec. VI. In addition, we calculate the high-frequency tail of the radio-frequency spectroscopy in Sec. VII. Finally, we summarize in Sec. VIII.

\section{MODEL AND NONINTERACTING PROPERTIES}

With the pseudopotential approximation, the effective 1D Hamiltonian with SOC is given by ( $\hbar=1$ throughout the paper) $[62,63,74-77]$

$$
\begin{aligned}
H= & \sum_{\sigma=\uparrow, \downarrow} \int d x \psi_{\sigma}^{\dagger}(x)\left(-\frac{1}{2 m} \frac{\partial^{2}}{\partial x^{2}}\right) \psi_{\sigma}(x) \\
& +\int d x \Omega\left[e^{i 2 k_{0} x} \psi_{\uparrow}^{\dagger}(x) \psi_{\downarrow}(x)+e^{-i 2 k_{0} x} \psi_{\downarrow}^{\dagger}(x) \psi_{\uparrow}(x)\right] \\
& +g_{1 \mathrm{D}} \int d x \psi_{\uparrow}^{\dagger}(x) \psi_{\downarrow}^{\dagger}(x) \psi_{\downarrow}(x) \psi_{\uparrow}(x)
\end{aligned}
$$

where $\psi_{\sigma}(x)$ is the field operator for the fermionic atoms, $x$ is the longitudinal atomic separation, the Fermi atoms in state $|\uparrow\rangle$ are coupled to state $|\downarrow\rangle$ by the Raman laser with the strength $\Omega=\Omega_{R} / 2, \Omega_{R}$ is the Rabi frequency, $2 k_{0}$ is the momentum transfer during the two-photon processes, and $g_{1 \mathrm{D}}$ is the even-wave coupling constant in 1D.

To remove the phase factor $e^{ \pm i 2 k_{0} x}$ in the second term of Eq. (1), we introduce two new atomic fields: $\psi_{\uparrow}(x)=$ $\psi_{\uparrow}(x) e^{i k_{0} x}$ and $\psi_{\downarrow}(x)=\psi_{\downarrow}(x) e^{-i k_{0} x}$. Then, we can write the single-particle part of the Hamiltonian in the momentum space: $H_{0}=\sum_{k} \Psi_{k}^{\dagger} \mathcal{H}_{0} \Psi_{k}$ with $\Psi_{k}=\left(a_{k, \uparrow}, a_{k, \downarrow}\right)^{T}$ and

$$
\mathcal{H}_{0}=\left(\begin{array}{cc}
\frac{\left(k+k_{0}\right)^{2}}{2 m} & \Omega \\
\Omega & \frac{\left(k-k_{0}\right)^{2}}{2 m}
\end{array}\right),
$$

where $a_{k, \sigma}$ is the field operator for the fermionic atoms in the momentum space.

Therefore, the inverse of the single-particle propagator matrix is given by $[68,79,80]$

$$
G^{-1}\left(q_{0}, k\right)=-i\left(\begin{array}{cc}
q_{0}+i 0^{+}-\frac{\left(k+k_{0}\right)^{2}}{2 m} & -\Omega \\
-\Omega & q_{0}+i 0^{+}-\frac{\left(k-k_{0}\right)^{2}}{2 m}
\end{array}\right),
$$

where $q_{0}$ is the total energy. Furthermore, we have

$$
\begin{aligned}
G\left(q_{0}, k\right) & =\int_{0}^{\infty} d t e^{i q_{0} t}\left\langle\mathcal{T} \Psi_{k}(t) \Psi_{k}^{\dagger}(0)\right\rangle \\
& =\left(\begin{array}{ll}
G_{\uparrow \uparrow}\left(q_{0}, k\right) & G_{\uparrow \downarrow}\left(q_{0}, k\right) \\
G_{\downarrow \uparrow}\left(q_{0}, k\right) & G_{\downarrow \downarrow}\left(q_{0}, k\right)
\end{array}\right),
\end{aligned}
$$

where $\mathcal{T}$ is the time-ordered operator and the elements are

$$
\begin{gathered}
G_{\uparrow \uparrow}\left(q_{0}, k\right)=\frac{i}{q_{0}-\frac{\left(k+k_{0}\right)^{2}}{2 m}+i 0^{+}-\frac{\Omega^{2}}{q_{0}-\frac{\left(k-k_{0}\right)^{2}}{2 m}+i 0^{+}}}, \\
G_{\uparrow \downarrow}\left(q_{0}, k\right)=G_{\downarrow \uparrow}\left(q_{0}, k\right) \\
=\frac{i \Omega}{\left[q_{0}-\frac{\left(k-k_{0}\right)^{2}}{2 m}+i 0^{+}\right]\left[q_{0}-\frac{\left(k+k_{0}\right)^{2}}{2 m}+i 0^{+}\right]-\Omega^{2}}, \\
G_{\downarrow \downarrow}\left(q_{0}, k\right)=\frac{i}{q_{0}-\frac{\left(k-k_{0}\right)^{2}}{2 m}+i 0^{+}-\frac{\Omega^{2}}{q_{0}-\frac{\left(k+k_{0}\right)^{2}}{2 m}+i 0^{+}}} .
\end{gathered}
$$

To calculate the Feynman diagrams for simplicity, the interacting part of the Hamiltonian can be written as

$$
H_{\text {int }}=g_{1 \mathrm{D}} \int d x\left[\frac{1}{2} \Psi^{\dagger}(x) \epsilon^{\dagger} \Psi^{\dagger T}(x)\right]\left[\frac{1}{2} \Psi^{T}(x) \epsilon \Psi(x)\right],
$$




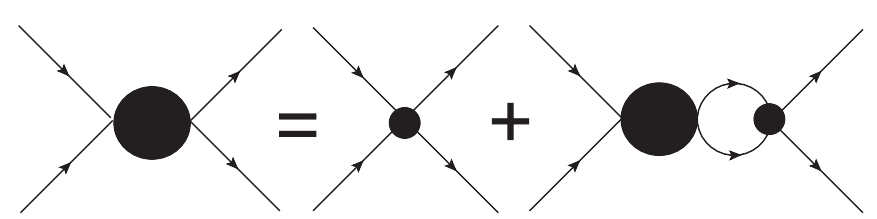

FIG. 1. Feynman diagrams for the $T$ matrix near a wide $s$-wave Feshbach resonance. The single line denotes the bare atom propagator matrix $G$. The black disk represents the $T$ matrix: $-i T_{s}$. The black dot represents the interaction vertex: $-i g_{1 \mathrm{D}}$.

where $\epsilon=-i \sigma_{y}$ is the two-by-two antisymmetric matrix $\Psi(x)=\left[\psi_{\uparrow}(x), \psi_{\downarrow}(x)\right]^{T}$ and we have

$$
\begin{aligned}
\frac{1}{2} \Psi^{T}(x) \epsilon \Psi(x) & =\frac{1}{2}\left[\psi_{\downarrow}(x) \psi_{\uparrow}(x)-\psi_{\uparrow}(x) \psi_{\downarrow}(x)\right] \\
& =\psi_{\downarrow}(x) \psi_{\uparrow}(x) .
\end{aligned}
$$

We can also write the interaction part of the Hamiltonian in the momentum space $[68,81-83]$,

$$
H_{\text {int }}=g_{1 \mathrm{D}} \sum_{k, k^{\prime}}\left(\frac{1}{2} \Psi_{k, k^{\prime}}^{\dagger} \epsilon^{\dagger} \Psi_{k, k^{\prime}}^{\dagger T}\right)\left(\frac{1}{2} \Psi_{k, k^{\prime}}^{T} \epsilon \Psi_{k, k^{\prime}}\right),
$$

where $\Psi_{k, k^{\prime}}=\left(a_{k, \uparrow}, a_{k^{\prime}, \downarrow}\right)^{T}$ and we have

$$
\frac{1}{2} \Psi_{k, k^{\prime}}^{T} \in \Psi_{k, k^{\prime}}=\frac{1}{2}\left(a_{k^{\prime}, \downarrow} a_{k, \uparrow}-a_{k, \uparrow} a_{k^{\prime}, \downarrow}\right)=a_{k^{\prime}, \downarrow} a_{k, \uparrow} .
$$

\section{TWO-BODY PHYSICS}

The two-body scattering process can be described by the two-body scattering amplitude or the scattering phase shift which can be determined by the two-body $T$ matrix in physics. In order to show the bare coupling constant $g_{1 \mathrm{D}}$ for the evenwave case needs to be renormalized or not, it is necessary to calculate the two-body $T$ matrix at first. Because the $T$ matrix is a physical quantity describing effective interaction in the low-energy space irrelevant to short-range physics.

We consider finite total momentum $Q$ for each twobody pairing state so that an incoming state can be set as $\left|I_{s}\right\rangle=|Q / 2+k, \uparrow ; Q / 2-k, \downarrow\rangle$ with two fermions of different species having momenta $Q / 2+k$ and $Q / 2-k$ to an outgoing state $\left|O_{s}\right\rangle=\left|Q / 2+k^{\prime}, \uparrow ; Q / 2-k^{\prime}, \downarrow\right\rangle$ with two fermions having momenta $Q / 2+k^{\prime}$ and $Q / 2-k^{\prime}$.

As shown in Fig. 1, the two-body $T$ matrix is given by [84]

$$
-i T_{s}\left(q_{0}, Q\right)=\frac{-i g_{1 \mathrm{D}}}{1-\left(-i g_{1 \mathrm{D}}\right) \Pi_{s}\left(q_{0}, Q\right)},
$$

where the polarization bubble is given by Refs. [68,81-83] (the derivations are given in the Appendix),

$$
\begin{aligned}
\Pi_{s}\left(q_{0}, Q\right)= & \int \frac{d p d p_{0}}{(2 \pi)^{2}} \frac{1}{2} \operatorname{Tr}\left[G^{T}\left(p_{0}, Q / 2+p\right) \epsilon G\left(q_{0}-p_{0}, Q / 2-p\right) \epsilon^{\dagger}\right] \\
= & \int \frac{d p d p_{0}}{(2 \pi)^{2}} \frac{1}{2}\left[G_{\uparrow \uparrow}\left(p_{0}, Q / 2+p\right) G_{\downarrow \downarrow}\left(q_{0}-p_{0}, Q / 2-p\right)+G_{\downarrow \downarrow}\left(p_{0}, Q / 2+p\right) G_{\uparrow \uparrow}\left(q_{0}-p_{0}, Q / 2-p\right)\right. \\
& \left.-G_{\uparrow \downarrow}\left(p_{0}, Q / 2+p\right) G_{\downarrow \uparrow}\left(q_{0}-p_{0}, Q / 2-p\right)-G_{\downarrow \uparrow}\left(p_{0}, Q / 2+p\right) G_{\uparrow \downarrow}\left(q_{0}-p_{0}, Q / 2-p\right)\right],
\end{aligned}
$$

$q_{0}=Q^{2} /(4 m)+k^{2} / m$, and $\operatorname{Tr}$ denotes the trace over the spin degrees of freedom.

In the absence of the Raman coupling, the even-wave coupling constant is given by (the derivations are given in the Appendix)

$$
g_{1 \mathrm{D}}=-\frac{1}{m_{r} a_{1 \mathrm{D}}},
$$

where $m_{r}=m / 2$ is the reduced mass, the even-wave scattering length in $1 \mathrm{D}$ is given by [74]

$$
a_{1 \mathrm{D}}=-\frac{\ell_{\perp}^{2}}{2 a_{s}}+\frac{\mathcal{C} \ell_{\perp}}{2},
$$

$a_{s}$ is the three-dimensional scattering length, $\mathcal{C}=$ 1.4603, $\ell_{\perp}=\sqrt{2 /\left(m \omega_{\perp}\right)}$, and $\omega_{\perp}$ is the transverse trapping frequency.

Note that Eq. (15) can only be valid for either a broad Feshbach resonance or the field-free case. Furthermore, the confinement induced resonance should also be broad. Since the external confinement is never perfectly harmonic nor isotropic, the possibility of inelastic resonances is possible. For example, a splitting of confinement-induced resonances has been observed in an anisotropic quasi-1D gas of Cs atoms in experiment [73]. Later, a theoretical model of the inelastic confinement-induced resonance was presented to describe the experimental observations [78].

In the presence of the Raman fields, the polarization bubble with zero total momentum $(Q=0)$ can be calculated as

$$
\begin{aligned}
\Pi_{s}\left(E_{0}, 0\right)= & \frac{m^{3} \Omega^{2}}{2\left(m E_{0} k_{0}^{2}-k_{0}^{4}+m^{2} \Omega^{2}\right) \sqrt{m E_{0}+i 0^{+}-k_{0}^{2}}} \\
& +\frac{m k_{0}^{2}}{4}\left[\frac{\sqrt{m E_{0}+k_{0}^{2}+2 \sqrt{m E_{0} k_{0}^{2}+m^{2} \Omega^{2}}}}{m^{2} \Omega^{2}+k_{0}^{2}\left(m E_{0}+\sqrt{m E_{0} k_{0}^{2}+m^{2} \Omega^{2}}\right)}\right. \\
& \left.+\frac{\sqrt{m E_{0}+i 0^{+}+k_{0}^{2}-2 \sqrt{m E_{0} k_{0}^{2}+m^{2} \Omega^{2}}}}{m^{2} \Omega^{2}+k_{0}^{2}\left(m E_{0}-\sqrt{m E_{0} k_{0}^{2}+m^{2} \Omega^{2}}\right)}\right],
\end{aligned}
$$

where $E_{0}=k^{2} / m$ and the $+i 0^{+}$terms are needed when $E_{0}<k_{0}^{2} / m$ and $m E_{0}+k_{0}^{2}<$ $2 \sqrt{m E_{0} k_{0}^{2}+m^{2} \Omega^{2}}, \quad$ such as $\sqrt{m E_{0}+i 0^{+}-k_{0}^{2}}=$ 


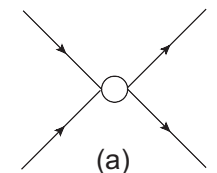

(a)

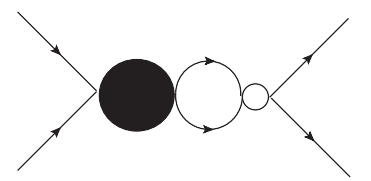

(c)

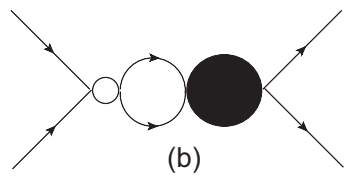

(b)

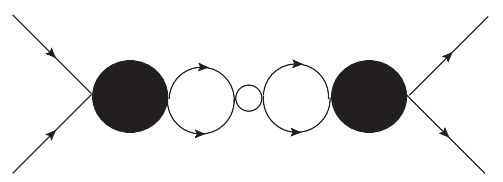

(d)
FIG. 2. Feynman diagrams for the matrix elements of the two-atom local operator $\psi_{\uparrow}^{\dagger}(R) \psi_{\downarrow}^{\dagger}(R) \psi_{\downarrow}(R) \psi_{\uparrow}(R)$ and its derivatives $\psi_{\uparrow}^{\dagger}(R) \psi_{\downarrow}^{\dagger}(R)\left[i \partial_{t}+\right.$ $\left.\partial_{R}^{2} /(4 m)\right]^{j} \psi_{\downarrow}(R) \psi_{\uparrow}(R), \psi_{\uparrow}^{\dagger}(R) \psi_{\downarrow}^{\dagger}(R)\left(-i \partial_{R}\right)^{n} \psi_{\downarrow}(R) \psi_{\uparrow}(R)$, and $\quad \psi_{\uparrow}^{\dagger}(R) \psi_{\downarrow}^{\dagger}(R)\left[i \partial_{t}+\partial_{R}^{2} /(4 m)\right]^{j}\left(-i \partial_{R}\right)^{n} \psi_{\downarrow}(R) \psi_{\uparrow}(R) \quad$ with $j, n=1-3, \ldots$. The open dots represent the operators.

$i \sqrt{k_{0}^{2}-m E_{0}}$ and $\sqrt{m E_{0}+i 0^{+}+k_{0}^{2}-2 \sqrt{m E_{0} k_{0}^{2}+m^{2} \Omega^{2}}}=$ $i \sqrt{2 \sqrt{m E_{0} k_{0}^{2}+m^{2} \Omega^{2}}-\left(m E_{0}+k_{0}^{2}\right)}$.

Note that the bubble (16) does not diverge and we do not need to have renormalization relation for $g_{1 \mathrm{D}}$.

The even-wave scattering amplitude in $1 \mathrm{D}$ can be written as $[20,74] f_{1 \mathrm{D}}(k)=-1 /\left(1+i \cot \delta_{k}\right)$, where $\delta_{k}$ is the scattering phase shift. With $T_{s}(k)=i k f_{1 \mathrm{D}}(k) / m_{r}$ and Eq. (16), one can get

$$
\cot \delta_{k}=\frac{k}{m_{r}}\left\{-\frac{1}{g_{1 \mathrm{D}}}-i\left[\Pi_{s}\left(E_{0}, 0\right)-\frac{m_{r}}{k}\right]\right\} .
$$

\section{EVEN-WAVE CONTACT}

To define the $1 \mathrm{D}$ even-wave contact, we need to give the adiabatic relation [20],

$$
\frac{C_{a}}{2 m} \equiv \frac{\partial E}{\partial a_{1 \mathrm{D}}}=\int d R\left\langle\frac{\partial \mathcal{H}}{\partial a_{1 \mathrm{D}}}\right\rangle
$$

where $E$ is the total energy of the many-body system, $\mathcal{H}$ is the density of the Hamiltonian (1), $C_{a}$ is the $1 \mathrm{D}$ even-wave contact, and we have used the following relations:

$\left\langle\frac{\partial \mathcal{H}}{\partial a_{1 \mathrm{D}}}\right\rangle=\left\langle\frac{\partial \mathcal{H}}{\partial g_{1 \mathrm{D}}}\right\rangle \frac{\partial g_{1 \mathrm{D}}}{\partial a_{1 \mathrm{D}}}=\frac{m}{2} g_{1 \mathrm{D}}^{2}\left\langle\psi_{\uparrow}^{\dagger}(R) \psi_{\downarrow}^{\dagger}(R) \psi_{\downarrow}(R) \psi_{\uparrow}(R)\right\rangle$.

Substituting Eq. (19) into (18), we get the expression for the contact $C_{a}$ as

$$
C_{a}=m^{2} g_{1 \mathrm{D}}^{2} \int d R\left\langle\psi_{\uparrow}^{\dagger}(R) \psi_{\downarrow}^{\dagger}(R) \psi_{\downarrow}(R) \psi_{\uparrow}(R)\right\rangle,
$$

where it is indicated that $C_{a}$ refers to the two-atom operator.

Furthermore, we calculate the expectation values of the two-atom operator $\left\langle O_{s}\left|\psi_{\uparrow}^{\dagger}(R) \psi_{\downarrow}^{\dagger}(R) \psi_{\downarrow}(R) \psi_{\uparrow}(R)\right| I_{s}\right\rangle$ as shown in Fig. 2 [17],

$$
\begin{aligned}
& \left\langle O_{s}\left|\psi_{\uparrow}^{\dagger}(R) \psi_{\downarrow}^{\dagger}(R) \psi_{\downarrow}(R) \psi_{\uparrow}(R)\right| I_{s}\right\rangle \\
& \quad=\sum_{j=a, b, c, d}\left\langle O_{s}\left|\psi_{\uparrow}^{\dagger}(R) \psi_{\downarrow}^{\dagger}(R) \psi_{\downarrow}(R) \psi_{\uparrow}(R)\right| I_{s}\right\rangle_{j} \\
& \quad=\left[1-i T_{s}\left(q_{0}, Q\right) \Pi_{s}\left(q_{0}, Q\right)\right]^{2} .
\end{aligned}
$$

Substituting Eq. (12) into (21), we have

$$
\left\langle O_{s}\left|\psi_{\uparrow}^{\dagger}(R) \psi_{\downarrow}^{\dagger}(R) \psi_{\downarrow}(R) \psi_{\uparrow}(R)\right| I_{s}\right\rangle=\frac{\left[T_{s}\left(q_{0}, Q\right)\right]^{2}}{g_{1 \mathrm{D}}^{2}} .
$$

Substituting Eq. (22) into (20), we get the even-wave contact as

$$
C_{a}=m^{2} \int d R\left[T_{s}\left(q_{0}, Q\right)\right]^{2}
$$

\section{UNIVERSAL RELATIONS}

\section{A. Adiabatic relations}

It is known that a contact can be defined to characterize the variation of energy as shown in Eq. (18). When SOC is present, there are two new parameters $k_{0}$ and $\Omega$. Similar to how we deal with the scattering length $a_{1 \mathrm{D}}$, one can define two new physical quantities $C_{\lambda}$ and $C_{\Omega}$ as

$$
\begin{aligned}
C_{\lambda} & \equiv \int d R\left\langle\frac{\partial \mathcal{H}}{\partial k_{0}}\right\rangle, \\
C_{\Omega} & \equiv \int d R\left\langle\frac{\partial \mathcal{H}}{\partial \Omega}\right\rangle .
\end{aligned}
$$

Here, $C_{\lambda}$ and $C_{\Omega}$ refer to only single-atom operators which give nonzero matrix elements in the single-atom sector. The momentum distribution under single-particle states is just a $\delta$ function so that $C_{\lambda}$ and $C_{\Omega}$ will not contribute to the largemomentum tail, which is different from $C_{a}$ [68]. However, both $k_{0}$ and $\Omega$ have nonzero energy scales so that they would appear in the pressure relation and viral theorem.

Note that the idea of the contact is that it describes coalescence of particles. $C_{\lambda}$ and $C_{\Omega}$ refer to single-particle properties, i.e., they are obviously nonzero even for a singleparticle or a noninteracting Fermi gas. Therefore, one cannot call them contacts, and we call them new physical quantities here.

\section{B. Pressure relation}

For a uniform gas, the pressure relation can be derived following the expression of the Helmholtz free-energy density $\mathcal{F}=F / L$ which can be expressed in terms of [5,20,21]

$$
\begin{aligned}
\mathcal{F}\left(T, n_{\uparrow}, n_{\downarrow}, a_{1 \mathrm{D}}, k_{0}, \Omega\right) \\
\quad=\frac{k_{F}^{3}}{2 m} f\left(\frac{2 m T}{k_{F}^{2}}, \frac{n_{\uparrow}}{k_{F}}, \frac{n_{\downarrow}}{k_{F}}, a_{1 \mathrm{D}} k_{F}, \frac{k_{0}}{k_{F}}, \frac{2 m \Omega}{k_{F}^{2}}\right),
\end{aligned}
$$

where $L$ is the length along the $x$ direction, $f$ is a dimensionless function, $T$ is the temperature, $n=n_{\uparrow}+n_{\downarrow}=k_{F} / \pi$ is the Fermi particle number density, and $k_{F}$ is the Fermi wave vector.

Equation (26) implies the scaling behavior of the Helmholtz free-energy density as follows:

$$
\begin{aligned}
& \tilde{\lambda}^{3} \mathcal{F}\left(T, n_{\uparrow}, n_{\downarrow}, a_{1 \mathrm{D}}, k_{0}, \Omega\right) \\
& \quad=\mathcal{F}\left(\tilde{\lambda}^{2} T, \tilde{\lambda} n_{\uparrow}, \tilde{\lambda} n_{\downarrow}, \tilde{\lambda}^{-1} a_{1 \mathrm{D}}, \tilde{\lambda} k_{0}, \tilde{\lambda}^{2} \Omega\right),
\end{aligned}
$$

where $\tilde{\lambda}$ is a dimensionless and arbitrary parameter. 
Taking the derivative of Eq. (27) with respect to $\tilde{\lambda}$ at $\tilde{\lambda}=1$, we have

$$
\begin{aligned}
3 \mathcal{F}= & \left(2 T \frac{\partial}{\partial T}+n_{\uparrow} \frac{\partial}{\partial n_{\uparrow}}+n_{\downarrow} \frac{\partial}{\partial n_{\downarrow}}-a_{1 \mathrm{D}} \frac{\partial}{\partial a_{1 \mathrm{D}}}\right. \\
& \left.+k_{0} \frac{\partial}{\partial k_{0}}+2 \Omega \frac{\partial}{\partial \Omega}\right) \mathcal{F} .
\end{aligned}
$$

Replacing the free-energy density $\mathcal{F}$ on the left side of Eq. (28) by $n_{\uparrow} \mu_{\uparrow}+n_{\downarrow} \mu_{\downarrow}-\mathcal{P}$ and substituting $S=-\partial F / \partial T$ and $\mu_{\sigma}=\partial F / \partial n_{\sigma}$ into Eq. (28), one gets

$$
\begin{aligned}
& 3\left(n_{\uparrow} \mu_{\uparrow}+n_{\downarrow} \mu_{\downarrow}-\mathcal{P}\right) \\
& \quad=-2 T S+n_{\uparrow} \mu_{\uparrow}+n_{\downarrow} \mu_{\downarrow}-a_{1 \mathrm{D}} \frac{\partial \mathcal{F}}{\partial a_{1 \mathrm{D}}}+k_{0} \frac{\partial \mathcal{F}}{\partial k_{0}}+2 \Omega \frac{\partial \mathcal{F}}{\partial \Omega},
\end{aligned}
$$

where $\mathcal{P}$ is the pressure density, $S$ is the entropy, and $\mu_{\sigma}$ is the chemical potential with spin $\sigma$.

Using the adiabatic relations (18), (24), and (25), we can get the pressure relation as

$$
\mathcal{P}=2 \mathcal{E}+\frac{a_{1 \mathrm{D}} C_{a}}{2 m L}-\frac{k_{0} C_{\lambda}}{L}-\frac{2 \Omega C_{\Omega}}{L},
$$

where $\mathcal{E}=E / L$ is the energy density and we use $E=F+$ $T S$.

\section{Virial theorem}

For an atomic gas in a harmonic potential $V_{T}=m \omega^{2} x^{2} / 2$ with the trapping frequency $\omega$, the free energy can be expressed in terms of $[5,20,21]$

$$
\begin{aligned}
& F\left(T, \omega, a_{1 \mathrm{D}}, k_{0}, \Omega, N_{\uparrow}, N_{\downarrow}\right) \\
& \quad=\omega \tilde{f}\left(T / \omega, \omega / \omega, a_{1 \mathrm{D}} / a_{\mathrm{ho}}, k_{0} a_{\mathrm{ho}}, \Omega / \omega, N_{\uparrow}, N_{\downarrow}\right),
\end{aligned}
$$

where $N=N_{\uparrow}+N_{\downarrow}$ is the particle number, $a_{\mathrm{ho}}=\sqrt{2 /(m \omega)}$ is the harmonic-oscillator length and the dimensionless function $\tilde{f}$ is dependent on the dimensionless ratios $T / \omega, a_{1 D} / a_{\mathrm{ho}}, k_{0} a_{\mathrm{ho}}, \Omega / \omega$, and particle numbers $N_{\uparrow}$ and $N_{\downarrow}$.

With Eq. (31), we can get the scaling law,

$$
\begin{aligned}
\tilde{\lambda} F & \left(T, \omega, a_{1 \mathrm{D}}, k_{0}, \Omega, N_{\uparrow}, N_{\downarrow}\right) \\
& =F\left(\tilde{\lambda} T, \tilde{\lambda} \omega, \tilde{\lambda}^{-1 / 2} a_{1 \mathrm{D}}, \tilde{\lambda}^{1 / 2} k_{0}, \tilde{\lambda} \Omega, N_{\uparrow}, N_{\downarrow}\right),
\end{aligned}
$$

where $\tilde{\lambda}$ is a dimensionless and arbitrary parameter.

The derivative of Eq. (32) with respect to $\tilde{\lambda}$ at $\tilde{\lambda}=1$ gives

$$
F=\left(T \frac{\partial}{\partial T}+\omega \frac{\partial}{\partial \omega}-\frac{1}{2} a_{1 \mathrm{D}} \frac{\partial}{\partial a_{1 \mathrm{D}}}+\frac{1}{2} k_{0} \frac{\partial}{\partial k_{0}}+\Omega \frac{\partial}{\partial \Omega}\right) F .
$$

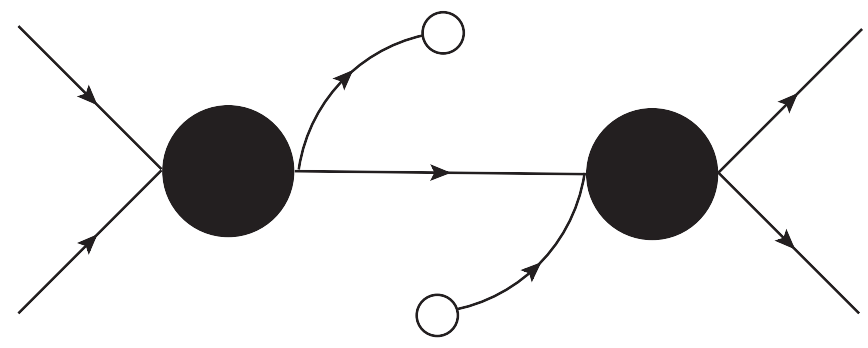

FIG. 3. Feynman diagram for the matrix elements of the operator $\psi_{\sigma}^{\dagger}\left(R+\frac{x}{2}\right) \psi_{\sigma^{\prime}}\left(R-\frac{x}{2}\right)$.

Substituting $E=F+T S$ and $S=-\partial F / \partial T$ into Eq. (33), one gets

$$
E=\left(\omega \frac{\partial}{\partial \omega}-\frac{1}{2} a_{1 \mathrm{D}} \frac{\partial}{\partial a_{1 \mathrm{D}}}+\frac{1}{2} k_{0} \frac{\partial}{\partial k_{0}}+\Omega \frac{\partial}{\partial \Omega}\right) E,
$$

which, together with the Hellmann-Feynman theorem and the adiabatic relations (18), (24), and (25), gives

$$
E=2\left\langle V_{T}\right\rangle-\frac{a_{1 \mathrm{D}} C_{a}}{4 m}+\frac{k_{0} C_{\lambda}}{2}+\Omega C_{\Omega}
$$

\section{LARGE-MOMENTUM TAIL}

In this section, we derive the tail of the momentum distribution for 1D fermions with SOC near a broad Feshbach resonance using the OPE method [4,5,21,22].

OPE is an ideal tool to explore the short-range physics. One can expand the product of two operators as

$$
\psi_{\sigma}^{\dagger}\left(R+\frac{x}{2}\right) \psi_{\sigma^{\prime}}\left(R-\frac{x}{2}\right)=\sum_{n} C_{n}(x) O_{n}(R),
$$

where $O_{n}(R)$ are the local operators and $C_{n}(x)$ are the shortdistance coefficients. $C_{n}(x)$ can be determined by calculating the matrix elements of the operators on both sides of Eq. (36) in the two-body state $\left|Q / 2+k, \sigma ; Q / 2-k, \sigma^{\prime}\right\rangle$.

By using the Fourier transformation on both sides of Eq. (36), we have the expression of momentum distribution as [5]

$$
n_{\sigma^{\prime} \sigma}(q)=\int \frac{d R}{L} \int d x e^{-i q x}\left\langle\psi_{\sigma}^{\dagger}\left(R+\frac{x}{2}\right) \psi_{\sigma^{\prime}}\left(R-\frac{x}{2}\right)\right\rangle,
$$

where $q$ is the relative momentum.

There are four types of diagrams which can be used to denote the operators on the left-hand side of the OPE equation (36). However, the only nonanalyticity comes from the diagram as shown in Fig. 3. Therefore, one can evaluate the diagram in Fig. 3 as (the derivations are given in the Appendix)

$$
\begin{gathered}
\left(\begin{array}{c}
\left\langle O_{s}\left|\psi_{\uparrow}^{\dagger}\left(R+\frac{x}{2}\right) \psi_{\uparrow}\left(R-\frac{x}{2}\right)\right| I_{s}\right\rangle_{d}\left\langle O_{s}\left|\psi_{\downarrow}^{\dagger}\left(R+\frac{x}{2}\right) \psi_{\uparrow}\left(R-\frac{x}{2}\right)\right| I_{s}\right\rangle_{d} \\
\left\langle O_{s}\left|\psi_{\uparrow}^{\dagger}\left(R+\frac{x}{2}\right) \psi_{\downarrow}\left(R-\frac{x}{2}\right)\right| I_{s}\right\rangle_{d}\left\langle O_{s}\left|\psi_{\downarrow}^{\dagger}\left(R+\frac{x}{2}\right) \psi_{\downarrow}\left(R-\frac{x}{2}\right)\right| I_{s}\right\rangle_{d}
\end{array}\right) \\
=\left[-i T_{s}\left(q_{0}, Q\right)\right]^{2} \int \frac{d p d p_{0}}{(2 \pi)^{2}} G\left(p_{0}, p\right) \epsilon^{\dagger} G^{T}\left(q_{0}-p_{0}, Q-p\right) \epsilon G\left(p_{0}, p\right) e^{-i p x}
\end{gathered}
$$


where $q_{0}=Q^{2} /(4 m)+k^{2} / m$ is the total energy, $Q$ is the center-of-mass momentum, and we use the Feynman rules for the operator vertices [5],

$$
\Psi^{\dagger}\left(R+\frac{x}{2}\right) \Psi\left(R-\frac{x}{2}\right) \sim \exp \left[-i p\left(R+\frac{x}{2}\right)\right] \exp \left[i p^{\prime}\left(R-\frac{x}{2}\right)\right]
$$

the incoming and outgoing momenta are $p$ and $p^{\prime}$ with $p=p^{\prime}$.

With the Fourier transforms, we get the momentum distribution matrix as

$$
n(q)=\left[-i T_{s}\left(q_{0}, Q\right)\right]^{2} \int_{-\infty}^{\infty} \frac{d p_{0}}{2 \pi} G\left(p_{0}, q\right) \epsilon^{\dagger} G^{T}\left(q_{0}-p_{0}, Q-q\right) \epsilon G\left(p_{0}, q\right)=\left(\begin{array}{ll}
n_{\uparrow \uparrow}(q) & n_{\uparrow \downarrow}(q) \\
n_{\downarrow \uparrow}(q) & n_{\downarrow \downarrow}(q)
\end{array}\right),
$$

where the analytical expressions for the elements of the matrix are shown in the Appendix.

Matching Eq. (40) with Eq. (22), we get the momentum distribution matrix in the large- $q$ limit up to the $q^{-8}$ order,

$$
\begin{aligned}
n(q)= & \frac{C_{a}}{q^{4} L}+\frac{2 \hat{\mathbf{q}} \cdot \mathbf{C}_{Q 1}-4 k_{0} C_{a} \sigma_{z}}{q^{5} L}+\frac{2 C_{R 1}+10 k_{0}^{2} C_{a}-10 k_{0} \hat{\mathbf{q}} \cdot \mathbf{C}_{Q 1} \sigma_{z}+5 C_{Q 2} / 2}{q^{6} L} \\
& +\frac{-12 k_{0} C_{R 1} \sigma_{z}-20 k_{0}^{3} C_{a} \sigma_{z}+6 \hat{\mathbf{q}} \cdot \mathbf{C}_{11}+30 k_{0}^{2} \hat{\mathbf{q}} \cdot \mathbf{C}_{Q 1}-15 k_{0} C_{Q 2} \sigma_{z}+5 \hat{\mathbf{q}} \cdot \mathbf{C}_{Q 3} / 2}{q^{7} L} \\
& +\frac{3 C_{R 2}+42 k_{0}^{2} C_{R 1}+35 k_{0}^{4} C_{a}-42 k_{0} \hat{\mathbf{q}} \cdot \mathbf{C}_{11} \sigma_{z}-70 k_{0}^{3} \hat{\mathbf{q}} \cdot \mathbf{C}_{Q 1} \sigma_{z}+21 C_{12} / 2+105 k_{0}^{2} C_{Q 2} / 2-35 k_{0} \hat{\mathbf{q}} \cdot \mathbf{C}_{Q 3} \sigma_{z} / 2+35 C_{Q 4} / 16}{q^{8} L},
\end{aligned}
$$

where we take the SOC parameters as perturbations since the strength of the SOC should be much smaller than the corresponding strength scale of the interatomic interactions, $\hat{\mathbf{q}}$ is the unit vector, and the new contacts are defined as

$$
\begin{gathered}
C_{R j}=m^{2+j} g_{1 \mathrm{D}}^{2} \int d R\left\langle\psi_{\uparrow}^{\dagger}(R) \psi_{\downarrow}^{\dagger}(R)\right. \\
\left.\times\left(i \partial_{t}+\frac{\partial_{R}^{2}}{4 m}\right)^{j} \psi_{\downarrow}(R) \psi_{\uparrow}(R)\right\rangle, \\
C_{Q n}=m^{2} g_{1 \mathrm{D}}^{2} \int d R\left\langle\psi_{\uparrow}^{\dagger}(R) \psi_{\downarrow}^{\dagger}(R)\left(-i \partial_{R}\right)^{n} \psi_{\downarrow}(R) \psi_{\uparrow}(R)\right\rangle, \\
C_{j n}=m^{2+j} g_{1 \mathrm{D}}^{2} \int d R\left\langle\psi_{\uparrow}^{\dagger}(R) \psi_{\downarrow}^{\dagger}(R)\right. \\
\left.\times\left(i \partial_{t}+\frac{\partial_{R}^{2}}{4 m}\right)^{j}\left(-i \partial_{R}\right)^{n} \psi_{\downarrow}(R) \psi_{\uparrow}(R)\right\rangle,
\end{gathered}
$$

$j, n=1-3, \ldots$. Note that, if $n$ is an odd number, the corresponding contact is a vector.

Different from $C_{\lambda}$ and $C_{\Omega}, C_{R j}$ refers to the effective range (as well as higher-order terms in the cotangent of the phase-shift expansion) which we do not discuss in our singlechannel model Hamiltonian (1), $C_{Q n}$ refers to the center-ofmass momentum, and $C_{j n}$ refers to both of the effective range (as well as higher-order terms in the cotangent of the phase shift expansion) and center-of-mass momentum.

In the laboratory frame, the single-particle Hamiltonian is given by $[62,63]$

$$
\mathcal{H}_{\mathrm{lab}, 0}=\left(\begin{array}{cc}
\frac{k^{2}}{2 m} & \Omega e^{i 2 k_{0} x} \\
\Omega e^{-i 2 k_{0} x} & \frac{k^{2}}{2 m}
\end{array}\right) .
$$

By transforming $n_{\uparrow \uparrow}(q)$ to $n_{\uparrow \uparrow}\left(q-k_{0}\right)$ and $n_{\downarrow \downarrow}(q)$ to $n_{\downarrow \downarrow}(q+$ $k_{0}$ ), the momentum distribution can go back to the laboratory frame as discussed in Ref. [68]. Furthermore, the diagonal elements of the momentum distribution matrix in the laboratory frame are calculated as

$$
\begin{aligned}
n_{\mathrm{lab}}(q)= & \frac{C_{a}}{q^{4} L}+\frac{2 \hat{\mathbf{q}} \cdot \mathbf{C}_{Q 1}}{q^{5} L}+\frac{4 C_{R 1}+5 C_{Q 2}}{2 q^{6} L} \\
& +\frac{12 \hat{\mathbf{q}} \cdot \mathbf{C}_{11}+5 \hat{\mathbf{q}} \cdot \mathbf{C}_{Q 3}}{2 q^{7} L}+\frac{48 C_{R 2}+168 C_{12}+35 C_{Q 4}}{16 q^{8} L} .
\end{aligned}
$$

It shows an anisotropic behavior at the $q^{-5}$ and $q^{-7}$ tails due to the center-of-mass momentum.

Meanwhile, the spin-dependent momentum distribution matrix in the laboratory frame can be written as

$$
\begin{aligned}
n_{\text {lab }, \text { spin-dep }}(q)= & \left(-\frac{16 k_{0}^{2} m \Omega C_{a}}{q^{8} L}-\frac{56 k_{0}^{2} m \Omega \hat{\mathbf{q}} \cdot \mathbf{C}_{Q 1}}{q^{9} L}\right) \sigma_{x} \\
& -\frac{16 k_{0} m^{2} \Omega^{2} C_{a}}{q^{9} L} \sigma_{z} .
\end{aligned}
$$

The diagonal elements of the momentum distribution matrix provide the expectation value of the atomic number with either spin $\uparrow$ or spin $\downarrow$ with a certain relative momentum $q$. The off-diagonal elements indicate the mixing of different spins. From the above equation (47), it is found that the $q^{-8}$ tail in the off-diagonal terms of the momentum distribution matrix is dependent on the SOC parameters in the laboratory frame. This tail can be observed through time-of-flight measurement as a direct manifestation of the SOC effects on the many-body level.

\section{RADIO-FREQUENCY SPECTROSCOPY}

The radio-frequency spectroscopy can be used as an important experimental tool in cold atom systems, and it can be derived from the Raman spectroscopy with zero relative momentum between two Raman lasers. In order to get the 


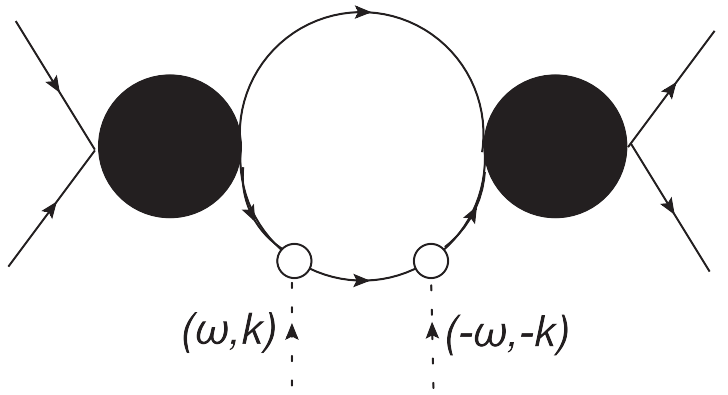

FIG. 4. Feynman diagram for the matrix element of $\int d t e^{i \omega t} \int d x e^{-i k x} \mathcal{T} O_{\sigma 3}(R+x, t) O_{\sigma^{\prime} 3}^{\dagger}(R, 0)(\sigma=\uparrow, \downarrow)$.

radio-frequency spectroscopy, we should derive the Raman spectroscopy first as follows. When the transfer momentum and frequency is larger compared to the many-body scale, the Raman spectroscopy can be related to the contacts.

We apply a Raman coupling with frequency $\omega$ and momentum $k$ which is applied to transfers fermions from the internal spin state $|\sigma\rangle(\sigma=\uparrow, \downarrow)$ into a third spin state $|3\rangle$. The Hamiltonian reads

$$
H_{c}=\sum_{\sigma} \Omega_{\sigma} \int d x e^{i(k x-\omega t)} O_{\sigma 3}(x, t)+\text { H.c. },
$$

where $O_{\sigma 3}(x, t) \equiv \psi_{3}^{\dagger}(x, t) \psi_{\sigma}(x, t), \Omega_{\sigma} \quad$ is the radiofrequency Rabi frequency determined by the strength of the radio-frequency signal and we assume $\omega>0$.

The transition rate function $R(\omega, k)$ is given by the Fermi golden rule, which is related to the imaginary part of the timeordered two-point correlation function $[85,86]$,

$$
\begin{aligned}
\Gamma_{\sigma \sigma^{\prime}}^{R}(\omega, k)= & \frac{1}{\pi} \operatorname{Im} \int d R \int d t e^{i \omega t} \int d x e^{-i k x} \\
& \times i\left\langle\mathcal{T} O_{\sigma 3}(R+x, t) O_{\sigma^{\prime} 3}^{\dagger}(R, 0)\right\rangle .
\end{aligned}
$$

Explicitly, we have the transition rate function $R(\omega, k)$,

$$
R(\omega, k)=2 \pi \sum_{\sigma \sigma^{\prime}} \Omega_{\sigma} \Omega_{\sigma^{\prime}}^{*} \Gamma_{\sigma \sigma^{\prime}}^{R}(\omega, k)
$$

Furthermore, we can evaluate the diagram in Fig. 4 as

$$
\begin{aligned}
\Gamma_{\sigma \sigma^{\prime}}^{R}(\omega, k) \\
=\frac{1}{\pi} \operatorname{Im} i\left[-i T_{s}\left(E_{0}, Q\right)\right]^{2} \\
\quad \times \int \frac{d p d p_{0}}{(2 \pi)^{2}} G_{0}\left(p_{0}+\omega, p+k\right) \\
\quad \times\left[G\left(p_{0}, p\right) \epsilon^{\dagger} G^{T}\left(E_{0}-p_{0}, Q-p\right) \epsilon G\left(p_{0}, p\right)\right]_{\sigma \sigma^{\prime}},
\end{aligned}
$$

where $E_{0}=Q^{2} /(4 m)+q^{2} / m$ is the total energy, $Q$ is the total momentum, and we have defined $G_{0}\left(p_{0}, p\right)=i /\left[p_{0}-\right.$ $\left.p^{2} /(2 m)+i 0^{+}\right]$.

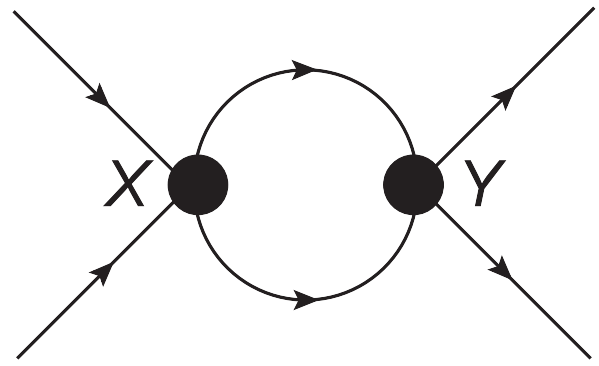

FIG. 5. Feynman diagram for the polarization bubble $\Pi_{s}$. Here, $X=\left(x_{1}, t_{1}\right)$ and $Y=\left(x_{2}, t_{2}\right)$.

Matching Eq. (49) with Eq. (22) and taking the limit of $k=$ 0 , we have the radio-frequency spectral $\Gamma_{\sigma \sigma^{\prime}}^{\mathrm{rf}}(\omega)=\Gamma_{\sigma \sigma^{\prime}}^{R}(\omega, 0)$ in the high-frequency limit,

$$
\begin{aligned}
\Gamma^{\mathrm{rf}}(\omega)= & \frac{m}{2 \pi}\left[\frac{C_{a}}{(m \omega)^{5 / 2}}+\frac{25 k_{0}^{2} C_{a}-4 C_{R 1}-10 k_{0} \hat{\mathbf{q}} \cdot \mathbf{C}_{Q 1} \sigma_{z}}{8(m \omega)^{7 / 2}}\right] \\
& -\frac{5 m^{2} \Omega C_{a}}{4 \pi(m \omega)^{7 / 2}} \sigma_{x} .
\end{aligned}
$$

As shown in Eq. (52), it is found that the high-frequency radio-frequency spectrum contains $C_{a}, C_{R 1}$, and $\mathbf{C}_{Q 1}$ as expected. Especially, the presence of $\mathbf{C}_{Q 1}$ in the radio-frequency spectrum is due to the SOC effects.

\section{SUMMARY}

We study the 1D two-component Fermi gases with SOC using the single-channel model and discuss some universal behaviors near a broad Feshbach resonance. Through the variation of energy with respect to the SOC parameters, two new physical quantities are defined, the pressure relation, and the viral theorem are derived in terms of the new SOC physical quantities. Utilizing the technique of OPE, the tail of the momentum distribution matrix is obtained. The momentum distribution matrix shows a spin-dependent behavior due to the SOC, and it shows an anisotropic behavior at the $q^{-5}$ and $q^{-7}$ tails due to the center-of-mass momentum. In the laboratory frame, the $q^{-8}$ tail in the momentum distribution can be observed through time-of-flight measurement as a direct manifestation of the SOC effects on the many-body level. In addition, the presence of the contact related to the center-of-mass momentum in the radio-frequency spectral is also due to the SOC effects.

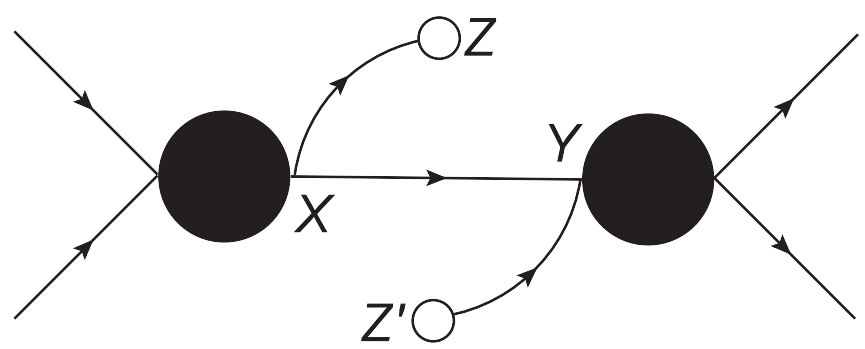

FIG. 6. Feynman diagram for the matrix of the operator $\Psi^{\dagger T}(Z) \Psi^{T}\left(Z^{\prime}\right)$. Here, $X=\left(x_{1}, t_{1}\right), Y=\left(x_{2}, t_{2}\right), Z=\left(x_{3}, t_{3}\right)$, and $Z^{\prime}=\left(x_{4}, t_{4}\right)$. 


\section{ACKNOWLEDGMENTS}

We acknowledge helpful discussions with Wei Yi, Xiaoling Cui, and Lianyi He. This work was supported by the National Natural Science Foundation of China (Grant No.
11404106). F.Q. acknowledges support from the project funded by the China Postdoctoral Science Foundation (Grant No. 2019M662150) and SUSTech Presidential Postdoctoral Fellowship.

\section{APPENDIX}

\section{Derivations of Eq. (13)}

The polarization bubble can be calculated by the diagram in Fig. 5 as

$$
\begin{aligned}
& \Pi_{s}\left(q_{0}, Q\right)=\int d X d Y\left\langle\mathcal{T}\left[\frac{1}{2} \Psi_{f}^{T}(X) \epsilon \Psi_{f}(X)\right]\left[\frac{1}{2} \Psi_{f}^{\dagger}(Y) \epsilon^{\dagger} \Psi_{f}^{\dagger T}(Y)\right]\right\rangle
\end{aligned}
$$

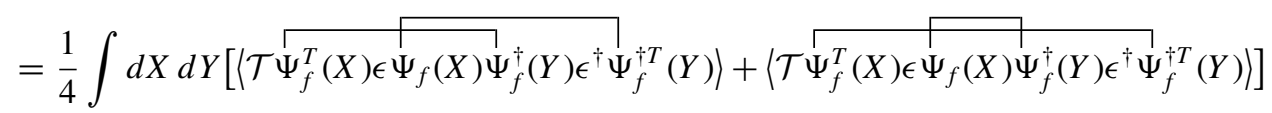

$$
\begin{aligned}
& =\frac{2}{4} \int d X d Y\langle\mathcal{T} \overbrace{f}^{T}(X) \epsilon \Psi_{f}(X) \Psi_{f}^{\dagger}(Y) \epsilon^{\dagger} \Psi_{f}^{\dagger T}(Y)\rangle \text { (two equivalent contractions), } \\
& =\frac{1}{2} \int d X d Y\left\langle\mathcal{T}\left[\Psi_{f}^{T}(X)\right]_{1 a}(\epsilon)_{a b}\left[\Psi_{f}(X)\right]_{b 1}\left[\Psi_{f}^{\dagger}(Y)\right]_{1 m}\left(\epsilon^{\dagger}\right)_{m n}\left[\Psi_{f}^{\dagger T}(Y)\right]_{n 1}\right\rangle \\
& =\frac{1}{2} \int d X d Y\left\langle\mathcal{T}\left[\Psi_{f}^{T}(X)\right]_{1 a}\left[\Psi_{f}^{\dagger T}(Y)\right]_{n 1}(\epsilon)_{a b}\left[\Psi_{f}(X)\right]_{b 1}\left[\Psi_{f}^{\dagger}(Y)\right]_{1 m}\left(\epsilon^{\dagger}\right)_{m n}\right\rangle \\
& =\frac{1}{2} \int d X d Y\left\langle\mathcal{T}\left[\Psi_{f}(X)\right]_{a 1}\left[\Psi_{f}^{\dagger}(Y)\right]_{1 n}\right\rangle(\epsilon)_{a b}[G(X-Y)]_{b m}\left(\epsilon^{\dagger}\right)_{m n} \\
& =\frac{1}{2} \int d X d Y[G(X-Y)]_{a n}(\epsilon)_{a b}[G(X-Y)]_{b m}\left(\epsilon^{\dagger}\right)_{m n} \\
& =\frac{1}{2} \int d X d Y\left[G^{T}(X-Y)\right]_{n a}(\epsilon)_{a b}[G(X-Y)]_{b m}\left(\epsilon^{\dagger}\right)_{m n} \\
& =\int d X d Y \frac{1}{2} \operatorname{Tr}\left[G^{T}(X-Y) \epsilon G(X-Y) \epsilon^{\dagger}\right],
\end{aligned}
$$

where $\int d X d Y=\frac{1}{2} \int d(X+Y) d(X-Y)$, we label the field operator $\Psi=\Psi_{s}+\Psi_{f}$ for outline as $\Psi_{s}$ and inner lines as $\Psi_{f}, \Psi_{f}=\left(\psi_{\uparrow}, \psi_{\downarrow}\right)^{T}, X=\left(x_{1}, t_{1}\right), Y=\left(x_{2}, t_{2}\right), q_{0}=Q^{2} /(4 m)+p^{2} / m$, and we use the definition $G(X-Y) \equiv$ $\left\langle\mathcal{T} \Psi_{f}(X) \Psi_{f}^{\dagger}(Y)\right\rangle$.

\section{Derivations of Eq. (14)}

In the absence of the Raman coupling, the polarization bubble is given by

$$
\Pi_{s}\left(q_{0}, Q\right)=\int \frac{d p d p_{0}}{(2 \pi)^{2}} \frac{i}{p_{0}-(Q / 2+p)^{2} /(2 m)+i 0^{+}} \frac{i}{q_{0}-p_{0}-(Q / 2-p)^{2} /(2 m)+i 0^{+}}=\frac{m}{2 k},
$$

where $q_{0}=Q^{2} /(4 m)+k^{2} / m$.

The even-wave scattering amplitude can be written as $[20,74] f_{1 \mathrm{D}}(k)=-1 /\left(1+i\right.$ cot $\left.\delta_{k}\right) \simeq-1 /\left(1+i k a_{1 \mathrm{D}}\right)$, where $\delta_{k}$ is the scattering phase shift. With $T_{s}(k)=i k f_{1 \mathrm{D}}(k) / m_{r}$, one can derive Eq. (14) in the main text.

\section{Derivations of Eq. (38)}

As shown in Fig. 6, the vacuum expectation of the operator $\Psi^{\dagger T}(Z) \Psi^{T}\left(Z^{\prime}\right)$ is calculated as follows:

$$
\begin{aligned}
\left\langle O_{s}\left|\Psi^{\dagger T}(Z) \Psi^{T}\left(Z^{\prime}\right)\right| I_{s}\right\rangle_{d} & \\
= & \left(\begin{array}{ll}
\left\langle O_{s}\left|\psi_{\uparrow}^{\dagger}(Z) \psi_{\uparrow}\left(Z^{\prime}\right)\right| I_{s}\right\rangle_{d} & \left\langle O_{s}\left|\psi_{\uparrow}^{\dagger}(Z) \psi_{\downarrow}\left(Z^{\prime}\right)\right| I_{s}\right\rangle_{d} \\
\left\langle O_{s}\left|\psi_{\downarrow}^{\dagger}(Z) \psi_{\uparrow}\left(Z^{\prime}\right)\right| I_{s}\right\rangle_{d} & \left\langle O_{s}\left|\psi_{\downarrow}^{\dagger}(Z) \psi_{\downarrow}\left(Z^{\prime}\right)\right| I_{s}\right\rangle_{d}
\end{array}\right) \\
& =\left[-i T_{s}\left(q_{0}, Q\right)\right]^{2} \int d X d Y\left\langle\mathcal{T} \Psi_{f}^{\dagger T}(Z)\left[\frac{1}{2} \Psi_{f}^{T}(X) \epsilon \Psi_{f}(X)\right]\left[\frac{1}{2} \Psi_{f}^{\dagger}(Y) \epsilon^{\dagger} \Psi_{f}^{\dagger T}(Y)\right] \Psi_{f}^{T}\left(Z^{\prime}\right)\right\rangle
\end{aligned}
$$




$$
\begin{aligned}
= & \frac{\left[-i T_{s}\left(q_{0}, Q\right)\right]^{2}}{4} \int d X d Y\left[\left\langle\mathcal{T} \Psi_{f}^{\dagger T}(Z) \Psi_{f}^{T}(X) \epsilon \Psi_{f}(X) \Psi_{f}^{\dagger}(Y) \epsilon^{\dagger} \Psi_{f}^{\dagger T}(Y) \Psi_{f}^{T}\left(Z^{\prime}\right)\right\rangle\right. \\
& +\left\langle\mathcal{T} \Psi_{f}^{\dagger T}(Z) \Psi_{f}^{T}(X) \epsilon \Psi_{f}(X) \Psi_{f}^{\dagger}(Y) \epsilon^{\dagger} \Psi_{f}^{\dagger T}(Y) \Psi_{f}^{T}\left(Z^{\prime}\right)\right\rangle+\left\langle\mathcal{T} \Psi_{f}^{\dagger T}(Z) \Psi_{f}^{T}(X) \epsilon \Psi_{f}(X) \Psi_{f}^{\dagger}(Y) \epsilon^{\dagger} \Psi_{f}^{\dagger T}(Y) \Psi_{f}^{T}\left(Z^{\prime}\right)\right\rangle \\
& \left.+\left\langle\mathcal{T} \Psi_{f}^{\dagger T}(Z) \Psi_{f}^{T}(X) \epsilon \Psi_{f}(X) \Psi_{f}^{\dagger}(Y) \epsilon^{\dagger} \Psi_{f}^{\dagger T}(Y) \Psi_{f}^{T}\left(Z^{\prime}\right)\right\rangle\right] \\
= & {\left[-i T_{s}\left(q_{0}, Q\right)\right]^{2} \int d X d Y\left\langle\mathcal{T} \Psi_{f}^{\dagger T}(Z) \Psi_{f}^{T}(X) \epsilon \Psi_{f}(X) \Psi_{f}^{\dagger}(Y) \epsilon^{\dagger} \Psi_{f}^{\dagger T}(Y) \Psi_{f}^{T}\left(Z^{\prime}\right)\right\rangle \text { (four equivalent contractions), } } \\
= & {\left[-i T_{s}\left(q_{0}, Q\right)\right]^{2} \int d X d Y\left\langle\mathcal{T}\left[\Psi_{f}^{\dagger T}(Z)\right]_{j 1}\left[\Psi_{f}^{T}(X)\right]_{1 m}(\epsilon)_{m n}\left[\Psi_{f}(X)\right]_{n 1}\left[\Psi_{f}^{\dagger}(Y)\right]_{1 a}\left(\epsilon^{\dagger}\right)_{a b}\left[\Psi_{f}^{\dagger T}(Y)\right]_{b 1}\left[\Psi_{f}^{T}\left(Z^{\prime}\right)\right]_{1 j}\right\rangle } \\
= & {\left[-i T_{s}\left(q_{0}, Q\right)\right]^{2} \int d X d Y\left\langle\mathcal{T}\left[\Psi_{f}(X)\right]_{m 1}\left[\Psi_{f}^{\dagger}(Z)\right]_{1 j}(\epsilon)_{m n}\left[\Psi_{f}(X]_{n 1}\left[\Psi_{f}^{\dagger}(Y)\right]_{1 a}\left(\epsilon^{\dagger}\right)_{a b}\left[\Psi_{f}\left(Z^{\prime}\right)\right]_{j 1}\left[\Psi_{f}^{\dagger}(Y)\right]_{1 b}\right\rangle\right.} \\
= & {\left[-i T_{s}\left(q_{0}, Q\right)\right]^{2} \int d X d Y[G(X-Z)]_{m j}(\epsilon)_{m n}[G(X-Y)]_{n a}\left(\epsilon^{\dagger}\right)_{a b}\left[G\left(Z^{\prime}-Y\right)\right]_{j b} } \\
= & {\left[-i T_{s}\left(q_{0}, Q\right)\right]^{2} \int d X d Y\left[G^{T}(X-Z)\right]_{j m}(\epsilon)_{m n}[G(X-Y)]_{n a}\left(\epsilon^{\dagger}\right)_{a b}\left[G^{T}\left(Z^{\prime}-Y\right)\right]_{b j} } \\
= & {\left[-i T_{s}\left(q_{0}, Q\right)\right]^{2} \int d X d Y G^{T}(X-Z) \epsilon G(X-Y) \epsilon^{\dagger} G^{T}\left(Z^{\prime}-Y\right) . }
\end{aligned}
$$

Therefore, we have

$$
\begin{aligned}
& \left(\begin{array}{ll}
\left\langle O_{s}\left|\psi_{\uparrow}^{\dagger}(Z) \psi_{\uparrow}\left(Z^{\prime}\right)\right| I_{S}\right\rangle_{d} & \left\langle O_{s}\left|\psi_{\uparrow}^{\dagger}(Z) \psi_{\downarrow}\left(Z^{\prime}\right)\right| I_{s}\right\rangle_{d} \\
\left\langle O_{s}\left|\psi_{\downarrow}^{\dagger}(Z) \psi_{\uparrow}\left(Z^{\prime}\right)\right| I_{s}\right\rangle_{d} & \left\langle O_{s}\left|\psi_{\downarrow}^{\dagger}(Z) \psi_{\downarrow}\left(Z^{\prime}\right)\right| I_{s}\right\rangle_{d}
\end{array}\right)^{T} \\
& \quad=\left[\left\langle O_{s}\left|\Psi^{\dagger T}(Z) \Psi^{T}\left(Z^{\prime}\right)\right| I_{s}\right\rangle_{d}\right]^{T}=\left[-i T_{s}\left(q_{0}, Q\right)\right]^{2} \int d X d Y G(X-Z) \epsilon^{\dagger} G^{T}(X-Y) \epsilon G\left(Z^{\prime}-Y\right) .
\end{aligned}
$$

\section{Elements of the momentum distribution matrix Eq. (40)}

$$
\begin{aligned}
& n_{\uparrow \uparrow}(q)=-\left[T_{s}\left(q_{0}, Q\right)\right]^{2} \int_{-\infty}^{\infty} \frac{d p_{0}}{2 \pi}\left[G_{\uparrow \uparrow}^{2}\left(p_{0}, q\right) G_{\downarrow \downarrow}\left(q_{0}-p_{0}, Q-q\right)+G_{\uparrow \uparrow}\left(q_{0}-p_{0}, Q-q\right) G_{\uparrow \downarrow}\left(p_{0}, q\right) G_{\downarrow \uparrow}\left(p_{0}, q\right)\right. \\
&\left.-G_{\uparrow \uparrow}\left(p_{0}, q\right) G_{\uparrow \downarrow}\left(p_{0}, q\right) G_{\downarrow \uparrow}\left(q_{0}-p_{0}, Q-q\right)-G_{\uparrow \uparrow}\left(p_{0}, q\right) G_{\downarrow \uparrow}\left(p_{0}, q\right) G_{\uparrow \downarrow}\left(q_{0}-p_{0}, Q-q\right)\right], \\
& n_{\uparrow \downarrow}(q)=-\left[T_{s}\left(q_{0}, Q\right)\right]^{2} \int_{-\infty}^{\infty} \frac{d p_{0}}{2 \pi}\left[G_{\uparrow \uparrow}\left(p_{0}, q\right) G_{\downarrow \downarrow}\left(q_{0}-p_{0}, Q-q\right) G_{\uparrow \downarrow}\left(p_{0}, q\right)\right. \\
&+G_{\uparrow \uparrow}\left(q_{0}-p_{0}, Q-q\right) G_{\downarrow \downarrow}\left(p_{0}, q\right) G_{\uparrow \downarrow}\left(p_{0}, q\right) \\
&\left.\quad-G_{\uparrow \uparrow}\left(p_{0}, q\right) G_{\downarrow \downarrow}\left(p_{0}, q\right) G_{\uparrow \downarrow}\left(q_{0}-p_{0}, Q-q\right)-G_{\uparrow \downarrow}^{2}\left(p_{0}, q\right) G_{\downarrow \uparrow}\left(q_{0}-p_{0}, Q-q\right)\right], \\
& n_{\downarrow \uparrow}(q)=-\left[T_{s}\left(q_{0}, Q\right)\right]^{2} \int_{-\infty}^{\infty} \frac{d p_{0}}{2 \pi}\left[G_{\uparrow \uparrow}\left(p_{0}, q\right) G_{\downarrow \downarrow}\left(q_{0}-p_{0}, Q-q\right) G_{\downarrow \uparrow}\left(p_{0}, q\right)\right. \\
&+G_{\uparrow \uparrow}\left(q_{0}-p_{0}, Q-q\right) G_{\downarrow \downarrow}\left(p_{0}, q\right) G_{\downarrow \uparrow}\left(p_{0}, q\right) \\
&\left.-G_{\uparrow \uparrow}\left(p_{0}, q\right) G_{\downarrow \downarrow}\left(p_{0}, q\right) G_{\downarrow \uparrow}\left(q_{0}-p_{0}, Q-q\right)-G_{\downarrow \uparrow}^{2}\left(p_{0}, q\right) G_{\uparrow \downarrow}\left(q_{0}-p_{0}, Q-q\right)\right],
\end{aligned}
$$

$n_{\downarrow \downarrow}(q)=-\left[T_{s}\left(q_{0}, Q\right)\right]^{2} \int_{-\infty}^{\infty} \frac{d p_{0}}{2 \pi}\left[G_{\downarrow \downarrow}^{2}\left(p_{0}, q\right) G_{\uparrow \uparrow}\left(q_{0}-p_{0}, Q-q\right)+G_{\downarrow \downarrow}\left(q_{0}-p_{0}, Q-q\right) G_{\downarrow \uparrow}\left(p_{0}, q\right) G_{\uparrow \downarrow}\left(p_{0}, q\right)\right.$$$
\left.-G_{\downarrow \downarrow}\left(p_{0}, q\right) G_{\uparrow \downarrow}\left(p_{0}, q\right) G_{\downarrow \uparrow}\left(q_{0}-p_{0}, Q-q\right)-G_{\downarrow \downarrow}\left(p_{0}, q\right) G_{\downarrow \uparrow}\left(p_{0}, q\right) G_{\uparrow \downarrow}\left(q_{0}-p_{0}, Q-q\right)\right] .
$$ 


\section{Perturbations of the elements of the single-particle propagator matrix}

We use the expansions with a small $\Omega$ as a perturbation,

$$
\begin{gathered}
G_{\uparrow \uparrow}\left(p_{0}, q\right) \approx \frac{i}{p_{0}-\frac{\left(q+k_{0}\right)^{2}}{2 m}+i 0^{+}}\left\{1+\frac{\Omega^{2}}{\left[p_{0}-\frac{\left(q+k_{0}\right)^{2}}{2 m}+i 0^{+}\right]\left[p_{0}-\frac{\left(q-k_{0}\right)^{2}}{2 m}+i 0^{+}\right]}\right\}, \\
G_{\uparrow \downarrow}\left(p_{0}, q\right)=G_{\downarrow \uparrow}\left(p_{0}, q\right) \approx \frac{\Omega^{2}}{\left[p_{0}-\frac{\left(q-k_{0}\right)^{2}}{2 m}+i 0^{+}\right]\left[p_{0}-\frac{\left(q+k_{0}\right)^{2}}{2 m}+i 0^{+}\right]}\left\{1+\frac{\left(p^{2}\right.}{\left[p_{0}-\frac{\left(q-k_{0}\right)^{2}}{2 m}+i 0^{+}\right]\left[p_{0}-\frac{\left(q+k_{0}\right)^{2}}{2 m}+i 0^{+}\right]}\right\}, \\
G_{\downarrow \downarrow}\left(p_{0}, q\right) \approx \frac{\Omega^{2}}{p_{0}-\frac{\left(q-k_{0}\right)^{2}}{2 m}+i 0^{+}}\left\{1+\frac{(}{\left[p_{0}-\frac{\left(q-k_{0}\right)^{2}}{2 m}+i 0^{+}\right]\left[p_{0}-\frac{\left(q+k_{0}\right)^{2}}{2 m}+i 0^{+}\right]}\right\} .
\end{gathered}
$$

\section{One-dimensional Fourier transforms}

The 1D Fourier transforms are $[20,87]$

$$
\int d x|x|^{\alpha} e^{-i q x}=-2 \alpha ! \sin \left(\frac{\pi \alpha}{2}\right) \frac{1}{|q|^{\alpha+1}},
$$

with $\alpha$ as an odd number, and

$$
\int d x \operatorname{sgn}(x)|x|^{\beta} e^{-i q x}=-2 i \beta ! \cos \left(\frac{\pi \beta}{2}\right) \frac{\operatorname{sgn}(q)}{|q|^{\beta+1}},
$$

with $\beta$ as an even number and $\operatorname{sgn}(x)$ as the sign function.

[1] S. Tan, Energetics of a strongly correlated Fermi gas, Ann. Phys. (NY) 323, 2952 (2008).

[2] S. Tan, Large momentum part of a strongly correlated Fermi gas, Ann. Phys. (NY) 323, 2971 (2008).

[3] S. Tan, Generalized virial theorem and pressure relation for a strongly correlated Fermi gas, Ann. Phys. (NY) 323, 2987 (2008).

[4] E. Braaten and L. Platter, Exact Relations for a Strongly Interacting Fermi Gas from the Operator Product Expansion, Phys. Rev. Lett. 100, 205301 (2008).

[5] E. Braaten, D. Kang, and L. Platter, Universal relations for a strongly interacting Fermi gas near a Feshbach resonance, Phys. Rev. A 78, 053606 (2008).

[6] S. Zhang and A. J. Leggett, Universal properties of the ultracold Fermi gas, Phys. Rev. A 79, 023601 (2009).

[7] S. B. Emmons, D. Kang, and L. Platter, Operator product expansion beyond leading order for two-component fermions, Phys. Rev. A 94, 043615 (2016).

[8] J. T. Stewart, J. P. Gaebler, T. E. Drake, and D. S. Jin, Verification of Universal Relations in a Strongly Interacting Fermi Gas, Phys. Rev. Lett. 104, 235301 (2010).

[9] Y. Sagi, T. E. Drake, R. Paudel, and D. S. Jin, Measurement of the Homogeneous Contact of a Unitary Fermi Gas, Phys. Rev. Lett. 109, 220402 (2012).

[10] S. Hoinka, M. Lingham, K. Fenech, H. Hu, C. J. Vale, J. E. Drut, and S. Gandolfi, Precise Determination of the Structure Factor and Contact in a Unitary Fermi Gas, Phys. Rev. Lett. 110, 055305 (2013).

[11] B. Song, Y. Yan, C. He, Z. Ren, Q. Zhou, G.-B. Jo, Evidence for Bosonization in a three-dimensional gas of SU(N) fermions, arXiv:1912.12105.
[12] Z. Yu, J. H. Thywissen, and S. Zhang, Universal Relations for a Fermi Gas Close to a $p$-Wave Interaction Resonance, Phys. Rev. Lett. 115, 135304 (2015); 117, 019901(E) (2016).

[13] M.-Y. He, S.-L. Zhang, H. M. Chan, and Q. Zhou, Concept of a Contact Spectrum and its Applications in Atomic Quantum Hall States, Phys. Rev. Lett. 116, 045301 (2016).

[14] S.-G. Peng, X.-J. Liu, and H. Hu, Large-momentum distribution of a polarized Fermi gas and $p$-wave contacts, Phys. Rev. A 94, 063651 (2016).

[15] S. M. Yoshida and M. Ueda, Universal High-Momentum Asymptote and Thermodynamic Relations in a Spinless Fermi Gas with a Resonant $p$-Wave Interaction, Phys. Rev. Lett. 115, 135303 (2015).

[16] F. Qin, X. Cui, and W. Yi, Universal relations and normal phase of an ultracold Fermi gas with coexisting $s$ - and $p$-wave interactions, Phys. Rev. A 94, 063616 (2016).

[17] F. Qin, Universal relations and normal-state properties of a Fermi gas with laser-dressed mixed-partial-wave interactions, Phys. Rev. A 98, 053621 (2018).

[18] C. Luciuk, S. Trotzky, S. Smale, Z. Yu, S. Zhang, and J. H. Thywissen, Evidence for universal relations describing a gas with $p$-wave interactions, Nat. Phys. 12, 599 (2016).

[19] P. Zhang, S. Zhang, and Z. Yu, Effective theory and universal relations for Fermi gases near a $d$-wave interaction resonance, Phys. Rev. A 95, 043609 (2017).

[20] M. Barth and W. Zwerger, Tan relations in one dimension, Ann. Phys. (NY) 326, 2544 (2011).

[21] X. Cui, Universal one-dimensional atomic gases near odd-wave resonance, Phys. Rev. A 94, 043636 (2016). 
[22] X. Cui and H. Dong, High-momentum distribution with subleading $k^{-3}$ tail in the odd-wave interacting one-dimensional Fermi gases, Phys. Rev. A 94, 063650 (2016).

[23] O. I. Patu and A. Klumper, Universal Tan relations for quantum gases in one dimension, Phys. Rev. A 96, 063612 (2017).

[24] X. Yin, X.-W. Guan, Y. Zhang, H. Su, and S. Zhang, Momentum distribution and contacts of one-dimensional spinless Fermi gases with an attractive $p$-wave interaction, Phys. Rev. A 98, 023605 (2018).

[25] M. Valiente, N. T. Zinner, and K. Mølmer, Universal relations for the two-dimensional spin-1/2 Fermi gas with contact interactions, Phys. Rev. A 84, 063626 (2011).

[26] M. Valiente, N. T. Zinner, and Klaus Mølmer, Universal properties of Fermi gases in arbitrary dimensions, Phys. Rev. A 86, 043616 (2012).

[27] F. Werner and Y. Castin, General relations for quantum gases in two and three dimensions: Two-component fermions, Phys. Rev. A 86, 013626 (2012).

[28] F. Werner and Y. Castin, General relations for quantum gases in two and three dimensions. II. Bosons and mixtures, Phys. Rev. A 86, 053633 (2012).

[29] J. Hofmann, Quantum Anomaly, Universal Relations, and Breathing Mode of a Two-Dimensional Fermi Gas, Phys. Rev. Lett. 108, 185303 (2012).

[30] Y.-C. Zhang and S. Zhang, Strongly interacting $p$-wave Fermi gas in two dimensions: Universal relations and breathing mode, Phys. Rev. A 95, 023603 (2017).

[31] S.-G. Peng, Universal relations for a spin-polarized Fermi gas in two dimensions, J. Phys. A: Math. Theor. 52, 245302 (2019).

[32] M. He and Q. Zhou, s-wave contacts of quantum gases in quasione-dimensional and quasi-two-dimensional traps, Phys. Rev. A 100, 012701 (2019).

[33] E. Braaten, D. Kang, and L. Platter, Universal Relations for Identical Bosons from Three-Body Physics, Phys. Rev. Lett. 106, 153005 (2011).

[34] D. H. Smith, E. Braaten, D. Kang, and L. Platter, Two-Body and Three-Body Contacts for Identical Bosons Near Unitarity, Phys. Rev. Lett. 112, 110402 (2014).

[35] R. J. Fletcher, R. Lopes, J. Man, N. Navon, R. P. Smith, M. W. Zwierlein, and Z. Hadzibabic, Two- and three-body contacts in the unitary bose gas, Science 355, 377 (2017).

[36] P. Zhang and Z. Yu, Signature of the universal super Efimov effect: Three-body contact in two-dimensional Fermi gases, Phys. Rev. A 95, 033611 (2017).

[37] P. Zhang and Z. Yu, Universal three-body bound states in mixed dimensions beyond the Efimov paradigm, Phys. Rev. A 96, 030702(R) (2017).

[38] Y. Nishida, S. Moroz, and D. T. Son, Super Efimov Effect of Resonantly Interacting Fermions in Two Dimensions, Phys. Rev. Lett. 110, 235301 (2013).

[39] Y. Nishida, Semisuper Efimov Effect of Two-Dimensional Bosons at a Three-Body Resonance, Phys. Rev. Lett. 118, 230601 (2017).

[40] F. Qin, J. Jie, W. Yi, and G.-C. Guo, High-momentum tail and universal relations of a Fermi gas near a Raman-dressed Feshbach resonance, Phys. Rev. A 97, 033610 (2018).

[41] M. He, C. Lv, H.-Q. Lin, and Q. Zhou, Universal relations for ultracold reactive molecules, arXiv:2005.07861.
[42] S. M. Yoshida and M. Ueda, $p$-wave contact tensor: Universal properties of axisymmetry-broken $p$-wave Fermi gases, Phys. Rev. A 94, 033611 (2016).

[43] S.-L. Zhang, M. He, and Q. Zhou, Contact matrix in dilute quantum systems, Phys. Rev. A 95, 062702 (2017).

[44] R. Weiss, B. Bazak, and N. Barnea, Nuclear Neutron-Proton Contact and the Photoabsorption Cross Section, Phys. Rev. Lett. 114, 012501 (2015).

[45] R. Weiss, B. Bazak, and N. Barnea, Generalized nuclear contacts and momentum distributions, Phys. Rev. C 92, 054311 (2015).

[46] R. Weiss, B. Bazak, and N. Barnea, The generalized nuclear contact and its application to the photoabsorption cross-section, Eur. Phys. J. A 52, 92 (2016).

[47] R. Weiss and N. Barnea, Contact formalism for coupled channels, Phys. Rev. C 96, 041303(R) (2017).

[48] R. Weiss, E. Pazy, and N. Barnea, Short range correlations: The important role of few-body dynamics in many-body systems, Few-Body Syst. 58, 9 (2017).

[49] R. Weiss, R. Cruz-Torres, N. Barnea, E. Piasetzky, and O. Hen, The nuclear contacts and short range correlations in nuclei, Phys. Lett. B 780, 211 (2018).

[50] R. Weiss, I. Korover, E. Piasetzky, O. Hen, and N. Barnea, Energy and momentum dependence of nuclear short-range correlations - Spectral function, exclusive scattering experiments and the contact formalism, Phys. Lett. B 791, 242 (2019).

[51] J. Hofmann, M. Barth, and W. Zwerger, Short-distance properties of Coulomb systems, Phys. Rev. B 87, 235125 (2013).

[52] B. Bazak, M. Valiente, and N. Barnea, Universal short-range correlations in bosonic helium clusters, Phys. Rev. A 101, 010501 (2020).

[53] Y. J. Lin, K. Jimenez-Garcia, and I. B. Spielman, Spin-orbitcoupled Bose-Einstein condensates, Nature (London) 471, 83 (2011).

[54] P. J. Wang, Z. Q. Yu, Z. K. Fu, J. Miao, L. H. Huang, S. J. Chai, H. Zhai, and J. Zhang, Spin-Orbit Coupled Degenerate Fermi Gases, Phys. Rev. Lett. 109, 095301 (2012).

[55] L. W. Cheuk, A. T. Sommer, Z. Hadzibabic, T. Yefsah, W. S. Bakr, and M. W. Zwierlein, Spin-Injection Spectroscopy of a Spin-Orbit Coupled Fermi Gas, Phys. Rev. Lett. 109, 095302 (2012).

[56] J.-Y. Zhang, S.-C. Ji, Z. Chen, L. Zhang, Z.-D. Du, B. Yan, G.-S. Pan, B. Zhao, Y.-J. Deng, H. Zhai, S. Chen, and J.W. Pan, Collective Dipole Oscillations of a Spin-Orbit Coupled Bose-Einstein Condensate, Phys. Rev. Lett. 109, 115301 (2012).

[57] V. Galitski and I. B. Spielman, Spin-orbit coupling in quantum gases, Nature (London) 494, 49 (2013).

[58] B. Song, C. He, S. Zhang, E. Hajiyev, W. Huang, X.J. Liu, and G.-B. Jo, Spin-orbit-coupled two-electron Fermi gases of ytterbium atoms, Phys. Rev. A 94, 061604(R) (2016).

[59] S. Zhang, C. He, E. Hajiyev, Z. Ren, B. Song, and G.-B. Jo, Collective dipole oscillations of a spin-orbit coupled Fermi gas, Sci. Rep. 8, 18005 (2018).

[60] N. Goldman, G. Juzeliunas, P. Ohberg, and I. B. Spielman, Light-induced gauge fields for ultracold atoms, Rep. Prog. Phys. 77, 126401 (2014). 
[61] J. Zhang, H. Hu, X.-J. Liu, and H. Pu, Fermi gases with synthetic spin-orbit coupling, Annu. Rev. Cold At. Mol. 2, 81 (2014).

[62] H. Zhai, Spin-orbit coupled quantum gases, Int. J. Mod. Phys. B 26, 1230001 (2012).

[63] H. Zhai, Degenerate quantum gases with spin-orbit coupling: A review, Rep. Prog. Phys. 78, 026001 (2015).

[64] Y. Xu and C. Zhang, Topological Fulde-Ferrell superfluids of a spin-orbit coupled Fermi gas, Int. J. Mod. Phys. B 29, 1530001 (2015).

[65] W. Yi, W. Zhang, and X. Cui, Pairing superfluidity in spin-orbit coupled ultracold Fermi gases, Sci. China: Phys., Mech. Astron. 58, 1 (2015).

[66] S.-G. Peng, C.-X. Zhang, S. Tan, and K. Jiang, Contact Theory for Spin-Orbit-Coupled Fermi Gases, Phys. Rev. Lett. 120, 060408 (2018).

[67] J. Jie, R. Qi, and P. Zhang, Universal relations of ultracold Fermi gases with arbitrary spin-orbit coupling, Phys. Rev. A 97, 053602 (2018)

[68] P. Zhang and N. Sun, Universal relations for spin-orbit coupled Fermi gas near an $s$-wave resonance, Phys. Rev. A 97, 040701(R) (2018).

[69] C.-X. Zhang, S.-G. Peng, and K. Jiang, Universal relations for spin-orbit-coupled Fermi gases in two and three dimensions, Phys. Rev. A 101, 043616 (2020).

[70] C. A. Regal, M. Greiner, and D. S. Jin, Observation of Resonance Condensation of Fermionic Atom Pairs, Phys. Rev. Lett. 92, 040403 (2004).

[71] I. Bloch, J. Dalibard, and W. Zwerger, Many-body physics with ultracold gases, Rev. Mod. Phys. 80, 885 (2008).

[72] C. Chin, R. Grimm, P. Julienne, and E. Tiesinga, Feshbach resonances in ultracold gases, Rev. Mod. Phys. 82, 1225 (2010).

[73] E. Haller, M. J. Mark, R. Hart, J. G. Danzl, L. Reichsöllner, V. Melezhik, P. Schmelcher, and H.-C. Nägerl, ConfinementInduced Resonances in Low-Dimensional Quantum Systems, Phys. Rev. Lett. 104, 153203 (2010).

[74] M. Olshanii, Atomic Scattering in the Presence of an External Confinement and a Gas of Impenetrable Bosons, Phys. Rev. Lett. 81, 938 (1998).
[75] T. Bergeman, M. G. Moore, and M. Olshanii, Atom-Atom Scattering under Cylindrical Harmonic Confinement: Numerical and Analytic Studies of the Confinement Induced Resonance, Phys. Rev. Lett. 91, 163201 (2003).

[76] X. Cui, Quasi-one-dimensional atomic gases across wide and narrow confinement-induced resonances, Phys. Rev. A 86, 012705 (2012).

[77] F. Qin, J.-S. Pan, S. Wang, and G.-C. Guo, Width of the confinement-induced resonance in a quasi-one-dimensional trap with transverse anisotropy, Eur. Phys. J. D 71, 304 (2017).

[78] S. Sala, P.-I. Schneider, and A. Saenz, Inelastic ConfinementInduced Resonances in Low-Dimensional Quantum Systems, Phys. Rev. Lett. 109, 073201 (2012).

[79] L. He, H. Hu, and X.-J. Liu, Realizing Fulde-Ferrell Superfluids via a Dark-State Control of Feshbach Resonances, Phys. Rev. Lett. 120, 045302 (2018).

[80] H. T. C. Stoof, K. B. Gubbels, and D. B. M. Dickerscheid, Ultracold Quantum Fields (Springer, Dordrecht, The Netherlands, 2009).

[81] S.-K. Jian, Y.-F. Jiang, and H. Yao, Emergent Spacetime Supersymmetry in 3D Weyl Semimetals and 2D Dirac Semimetals, Phys. Rev. Lett. 114, 237001 (2015).

[82] R. Nandkishore, J. Maciejko, D. A. Huse, S. L. Sondhi, Superconductivity of disordered Dirac fermions, Phys. Rev. B 87, 174511 (2013).

[83] E.-G. Moon, C. Xu, Y. B. Kim, and L. Balents, Non-FermiLiquid and Topological States with Strong Spin-Orbit Coupling, Phys. Rev. Lett. 111, 206401 (2013).

[84] V. Gurarie and L. Radzihovsky, Resonantly-paired fermionic superfluids, Ann. Phys. (NY) 322, 2 (2007).

[85] E. Braaten, D. Kang, and L. Platter, Short-Time Operator Product Expansion for Rf Spectroscopy of A Strongly Interacting Fermi Gas, Phys. Rev. Lett. 104, 223004 (2010).

[86] J. Hofmann, Current response, structure factor and hydrodynamic quantities of a two- and three- dimensional fermi gas from the operator-product expansion, Phys. Rev. A 84, 043603 (2011).

[87] D. Kammler, A First Course in Fourier Analysis (Cambridge University Press, Cambridge, U.K., 2007). 